\title{
Challenges in Predictive Modeling of Single Pass Tangential Flow Filtration for Continuous Biomanufacturing
}

\author{
Madeline Fuchs ${ }^{1}$, Rajan Bhawnani ${ }^{1}$, Sobhana Alekhya Sripada ${ }^{1}$, Jessica Molek ${ }^{1}$, and Mehdi \\ Ghodbane $^{1}$ \\ ${ }^{1}$ GlaxoSmithKline USA
}

January 4, 2022

\begin{abstract}
Opportunities for process intensification and increased productivity have made the field of Continuous Biomanufacturing an area of high interest and active research. Within the purification train of producing biologics, Tangential Flow Filtration (TFF) is typically employed after chromatographic separations, to increase drug substance concentration, making the process more economical and further meeting dosage specifications. In a batch operation, concentration occurs via recirculation of the feed material where desired output concentration is attained through multiple pump-passes over the TFF membrane, while steadily excluding the buffer. Single-Pass Tangential Flow Filtration (SPTFF) enables continuity of this process by achieving similar concentration factors through a single - pass over these membranes while operating at low feed flow rates. Our work elucidates the development of a mechanistic process model to predict SPTFF performance across a relatively wide design space using a first principles approach. The developed model is found to be accurate for a range of high feed flow rates but is inaccurate at flow rates below $25 \mathrm{~L} / \mathrm{m} 2 / \mathrm{hr}$. At very low flow rates, small differences in the mass transfer coefficient have been observed to significantly alter the prediction of the retentate concentration. We thus describe the challenges in predictive process modeling of SPTFF in antibody biomanufacturing.
\end{abstract}

\section{Challenges in Predictive Modeling of Single Pass Tangential Flow Filtration for Continuous Biomanufacturing}

Madeline Fuchs ${ }^{1,4}+$, Rajan Bhawnani ${ }^{2,4}+$, Sobhana A. Sripada ${ }^{3,4}$, Jessica Molek $^{4,5}$, Mehdi Ghodbane ${ }^{4,+}$ 1 J. Crayton Pruitt Family Department of Biomedical Engineering, University of Florida, Gainesville FL, $U S A$

${ }^{2}$ Department of Chemical Engineering, University of Illinois at Chicago, Chicago IL, USA

3 Department of Chemical and Biomolecular Engineering, North Carolina State University, Raleigh NC, $U S A$

4 Downstream Process Development, GlaxoSmithKline, King of Prussia, PA, USA

${ }^{5}$ MSAT Specialty Large Molecule, GlaxoSmithKline, King of Prussia, PA, USA

+ Authors contributed equally

++ Corresponding author: Mehdi.x.Ghodbane@gsk.com

\section{Abstract:}

Opportunities for process intensification and increased productivity have made the field of Continuous Biomanufacturing an area of high interest and active research. Within the purification train of producing biologics, Tangential Flow Filtration (TFF) is typically employed after chromatographic separations, 
to increase drug substance concentration, making the process more economical and further meeting dosage specifications. In a batch operation, concentration occurs via recirculation of the feed material where desired output concentration is attained through multiple pump-passes over the TFF membrane, while steadily excluding the buffer. Single-Pass Tangential Flow Filtration (SPTFF) enables continuity of this process by achieving similar concentration factors through a single - pass over these membranes while operating at low feed flow rates.

Our work elucidates the development of a mechanistic process model to predict SPTFF performance across a relatively wide design space using a first principles approach. The developed model is found to be accurate for a range of high feed flow rates but is inaccurate at flow rates below $25 \mathrm{~L} / \mathrm{m}^{2} / \mathrm{hr}$. At very low flow rates, small differences in the mass transfer coefficient have been observed to significantly alter the prediction of the retentate concentration. We thus describe the challenges in predictive process modeling of SPTFF in antibody biomanufacturing.

Keywords: Antibody Concentration, Continuous Biomanufacturing, SPTFF, Ultrafiltration, Membrane Filtration

\section{Introduction}

Tangential flow filtration (TFF) is widely performed in biopharmaceutical downstream purification processes to concentrate proteins and perform buffer exchange (Foley, 2013; H. Lutz, 2015; Teske et al., 2010). An ultrafiltration membrane with a nominal molecular weight cutoff (3-5x smaller than the protein of interest) is typically employed to facilitate full retention of the protein, enabling buffer permeation (H. Lutz, 2015; Millipore, 1999). The retentate stream is recirculated into the feed vessel during the startup phase resulting in multiple passes through the filter assembly, to achieve the desired output concentration, making it an inherently batch process. Alternatively, this can be performed with the retentate stream directed into a separate collection vessel. This configuration, referred to as Single Pass Tangential Flow Filtration (SPTFF), has the product pass only once over the membranes in the unit process. To achieve target concentrations without recirculation, SPTFF can be performed at much lower flow rates than a traditional (recirculation enabled) TFF, resulting in increased residence time through the filter assembly and thus higher conversions leading to higher product concentrations (Dizon-Maspat et al., 2012; H. Lutz, 2015; Millipore, 1999; Teske et al., 2010).

SPTFF has been utilized in a variety of downstream processing applications. Volume reduction of in-process products can minimize intermediate hold-tank requirements, prevent facility fit deliberations, and decrease filter and column sizes (Dizon-Maspat et al., 2012; H. Lutz, 2015; Miranda \& Campos, 2002; Teske et al., 2010). In addition, it is difficult to accommodate a batch TFF into a continuous process due to retentate recirculation that may result in product accumulation upstream of the unit operation and disrupt product flow to the downstream operations. SPTFF is therefore an operation that can facilitate end-to-end continuous processing. However, using SPTFF in a truly continuous process to achieve a target product concentration is challenging due to flow rate constraints required to ensure continuity of the purification process. Therefore, development of an effective concentration step requires an in-depth understanding of the effect of filter configuration and input flow rate on output concentrations. Research is being increasingly conducted to understand these effects specifically (Thakur \& Rathore, 2021). The case of SPTFF is particularly challenging when compared to batch TFF, where targets can be relatively easily reached by operating in recirculation until the target concentration is reached.

In our studies, we develop and demonstrate the performance of a model capable of predicting the output concentration of the SPTFF ultrafiltration step at various operating parameters. Such an effort is expected to facilitate proper membrane sizing and configuration for a wide range of processes where input flow rate and concentration are fixed, with minimal experimental work. To this end, flux excursion experiments were performed to generate a data set to which the stagnant film model was applied, and additional experiments were performed to test the model and empirically fine - tune it. As described in the forthcoming sections, we observed model inaccuracies at low feed fluxes that led to the evaluation of maximum conversion across 
a single membrane. The behavior observed could not be accurately predicted by the stagnant film model, so the assumptions of the model in this low flux regime were analyzed. Through the course of this paper, we provide an overview of implementing the stagnant film model for SPTFF and highlight the limitations of this analysis.

\section{Materials and Methods}

All the experiments described in this paper were performed using $88 \mathrm{~cm}^{2}$ Millipore Ultracel Pellicon 3 CScreen cassettes with a nominal molecular weight cutoff of $30 \mathrm{kDa}$ (Cat. No P3C030C00). A monoclonal antibody $(147.8 \mathrm{kDa})$ at a concentration of $8 \mathrm{mg} / \mathrm{mL}$ - prepared by purifying harvested CHO cell culture through protein A chromatography, low $\mathrm{pH}$ viral inactivation, anion exchange chromatography, and viral nanofiltration, was used as the feed material. Various membrane configurations were formulated using diverter plates between individual membranes (Cat. No. XXSPTFF01). The orientation of the diverter plates dictated the series / parallel nature of the membrane configuration, as described in Millipore's TFF Operating Guide (Millipore, 1999).

Membranes were assembled in a Millipore Pellicon Mini Cassette Holder (Cat. No. XX42PMINI) and sanitized according to the manufacturer's guidelines. Briefly, sanitization was conducted with a flush volume of $20 \mathrm{~L} / \mathrm{m}^{2} 0.5 \mathrm{M} \mathrm{NaOH}$ at $4 \mathrm{~L} / \mathrm{m}^{2} / \mathrm{min}$. The system was held in $0.5 \mathrm{M} \mathrm{NaOH}$ for an hour and flushed using $0.1 \mathrm{M} \mathrm{NaOH}$ before storage. The system was restarted by re-equilibrating the system with appropriate buffers.

Pressure independent regions for operation were deduced through flux excursion studies using one $88 \mathrm{~cm}^{2}$ membrane. The design space used for conducting these experiments is listed in Table 1. Pressure was monitored at the feed inlet and retentate and permeate outlets using PressureMAT sensors (PendoTECH, PMAT4R).

Steady state was assumed to be achieved when the retentate concentration stabilized, which was measured using the SoloVPE instrument (C Technologies, Inc.). Once steady, the output concentration (CR) and permeate flux $(\mathrm{J})$ were recorded. TMP was controlled manually using a needle valve (Swagelok) at the retentate outlet. A predictive model was built using this flux excursion data according to the stagnant film model theory described in section 3 .

Two SPTFF experiments were performed to validate the model developed with a single membrane. The first experiment involved a three-membrane configuration with alternating diverter plates separating them into three stages of equal area. The TMP was controlled only across the final stage using an automated pinch valve (PendoTECH Throttle Valve PDKT-PVT-P). The inlet pressure to the first membrane was used as the feed pressure and the retentate pressure was measured at the final outlet of the filter assembly. Protein was fed into the system at 68 and $116 \mathrm{LMH}$ respectively. Retentate concentration at the outlet of the third stage was recorded as the steady state value. Retentate concentration was also measured at the first and second stages to collect additional data for understanding the system operation. Finally, the measurement of maximum conversion was performed by concentrating a feed at $5.4 \mathrm{~g} / \mathrm{L}$ using a single $88 \mathrm{~cm}^{2}$ membrane at various feed fluxes ranging from 5 to $60 \mathrm{LMH}$.

\section{Theory}

Tangential flow filtration is governed by stagnant film theory, which states that permeate flux is directly proportional to the concentration gradient. At a steady state, mass transfer of solute to the membrane surface is equal to diffusion away from the membrane, leading to a constant fluid boundary gel layer of a specific thickness and concentration. Mass transfer is assumed to occur across this fluid boundary layer (Zydney, 1997). Mass balance of this layer can be written as:

$J=-D \frac{\mathrm{dC}}{\mathrm{dz}}(3.1)$

Integrating equation (3.1) and applying boundary conditions, we obtain

$J=\frac{D}{\delta} \ln \frac{C w-C p}{C f-C p}$ 
The term $\frac{D}{\delta}$ is the mass transfer coefficient k. For an intact, retaining membrane, $\mathrm{C}_{\mathrm{p}}$ is zero, therefore simplifying equation 3.2 to:

$J=k \ln \frac{\mathrm{Cw}}{\mathrm{Cf}}(3.3)$

Permeate flux is also governed by the average differential pressure across the membrane, also known as the transmembrane pressure (TMP).

$T M P=\frac{P_{\text {Feed }}+P_{\text {Retentate }}}{2}-P_{\text {Permeate }}(3.4)$

Flux across the membrane can be increased by increasing the TMP until a critical pressure where mass transfer limits flux across the membrane. This region is known as the pressure independent region. Such limiting flux behavior is typically explained by two mechanisms: (1) an increase in resistance to flow and (2) an increase in osmotic pressure, both due to the tightly packed protein gel layer at the membrane surface. A fundamental limitation to flux in this regime can be understood as the mass transfer of protein away from the membrane, rather than pressure. To operate in such a range, the region of pressure independence is characterized through flux excursion experiments carried out at various feed flow rates and concentrations.

A plot of $J$ vs $\ln \left(C_{b}\right)$, where $C_{b}$ is the bulk feed concentration, in the pressure independent region at a particular flow rate results in a straight line with slope of $-\mathrm{k}$ and $\mathrm{x}$-intercept of $\ln \left(\mathrm{C}_{\mathrm{w}}\right)$. Data from each flow rate produces a line with a different slope, or mass transfer coefficient. The effect of feed flow rate on the mass transfer coefficient $\mathrm{k}$ is then characterized by using the Sherwood number.

$S h=\frac{\mathrm{kL}}{D}=\beta \cdot \operatorname{Re}^{a} \cdot \mathrm{Sc}^{b} \cdot\left(\frac{L}{D}\right)^{c} \cdot\left(\frac{\mu_{b}}{\mu_{w}}\right)^{d}(3.5)$

A common assumption to determine the mass transfer coefficient from the above equation is that the fluid properties at the membrane wall and in the bulk stay constant across flow rates (Teske et al., 2010). Therefore, all the terms in equation 3.5 except the Reynolds number are constant and the mass transfer coefficient at different flow rates can be expressed as:

$\frac{k_{1}}{k_{0}}=\left(\frac{Q_{1}}{Q_{0}}\right)^{a}(3.6)$

The value of ' $\mathrm{a}$ ' can be empirically determined resulting in a relationship between $\mathrm{k}$ and feed flow rate, which can be used to predict the conversion of a process at various bulk concentrations. The value of $\mathrm{C}_{\mathrm{w}}$ is calculated by taking the exponential of the $\mathrm{x}$-intercept in the $\mathrm{J}$ vs $\ln \left(\mathrm{C}_{\mathrm{b}}\right)$ plot. The conversion for $\mathrm{a}$ membrane separation process is defined as the ratio of the permeate flux to the feed flux.

$\varnothing=\frac{\mathrm{J} . \mathrm{A}}{q_{f}}=\frac{J}{Q}=\frac{k}{Q} \ln \left(\frac{C_{w}}{C_{b}}\right)$

The equation for the final retentate concentration is derived using mass balance:

$C_{R}=\frac{C_{f}}{1-\varnothing}(3.8)$

\section{Results and Discussion}

\section{Flux Excursions}

Flux excursion experiments were performed across a single $88 \mathrm{~cm}^{2}$ cassette by varying feed flow rate, TMP, and feed concentration, as described in Figure 1. These flux excursion experiments for developing SPTFF are identical to those required for batch TFF, except they are conducted at much lower feed fluxes. We observed that the permeate flux, J, was independent of TMP above 15 psi for all conditions. Due to the pressure drop across membrane cassettes, the TMP on the last membrane was slightly lower than the preceding membranes. For a multi-stage setup, the TMP across the final stage was controlled by an automated back pressure valve at the outlet of the final stage. Therefore, to ensure all stages operate in the pressure independent region, the TMP of the third stage was set to 15 psi. Operating at the edge of this pressure independent region maximized our permeate flux and the concentration factor while minimizing membrane fouling (van Reis et al., 1997; Zydney, 1997). Additionally, it implemented an ease of operation and process development, as well as reduced sensitivity to pressure fluctuations. 


\section{Stagnant Film Modeling - First Principles Approach Predictive Model Development}

To build the stagnant film model, the measured permeate flux at 15 psi for each condition was plotted against the natural log of feed concentration at each flow rate tested (Figure 2). The slope and intercepts were used to determine the mass transfer coefficient and wall concentration respectively at each feed flux. The Sherwood number approximation (Equation 3.5) was then used to make appropriate predictions of the mass transfer coefficient $\mathrm{k}$, using the stagnant film theory, simplified as equation 3.6, assuming constant bulk fluid properties. The values of $\mathrm{k}$ evaluated using equation 3.6 have been shown in Figure 3A. The initial properties $\left(\mathrm{Q}_{0}\right.$ and $\left.\mathrm{k}_{0}\right)$ were chosen from the data points generated from our experiments. For the analyses, we set $\mathrm{Q}_{0}$ to $82 \mathrm{LMH}$ and $\mathrm{k}_{0}$ to its corresponding mass transfer coefficient. The exponent ' $\mathrm{a}$ ' was determined empirically from the least-squared best-fit analytical value of the remaining flux excursion data (Teske et al., 2010).

This approach and model required approximating $\mathrm{k}$ and wall concentration $\left(\mathrm{C}_{\mathrm{w}}\right)$ to predict the overall conversion of the process (Equation 3.7). The $\mathrm{x}$-intercept from the $\mathrm{J}$ vs $\ln \left(\mathrm{C}_{\mathrm{w}}\right)$ plot was used as the value of the wall concentration. Based on the stagnant film theory, $\mathrm{C}_{\mathrm{w}}$ was assumed constant across all flow rates (Teske et al., 2010; Zydney, 1997). However, it was then observed that $\mathrm{C}_{\mathrm{w}}$ had an inverse linear relationship with feed flow rate, as shown in Figure 3B. One of the possible hypotheses for this observation is the fact that the SPTFF is operated at lower cross flow rates than batch TFF. This reduced shear appears to lead to higher deposition of concentrated material on the membrane surface.

Equation 4.1 is the complete stagnant film model for permeate flux across a single membrane at a given input concentration and flow rate.

$J=k_{o}\left(\frac{Q}{Q_{o}}\right)^{a} \ln \left(\frac{m Q+b}{C_{b}}\right)$

Where, $\mathrm{mQ}+\mathrm{b}$ is the linear fit of the $\mathrm{C}_{\mathrm{w}}$ vs. $\mathrm{Q}$ graph in Fig. 3B

This model, combined with equation 3.6 and 3.7, was then extended to a multi-stage SPTFF by using the output concentration and flow rate of one stage as the input to the next. This led to the construction of a predictive model that could calculate output concentration based on input flow rate, feed concentration, number of stages, and relative surface area of those stages. Figure 4 shows the predicted output concentration curves for a configuration with stages of the same relative area and a feed concentration of $8.2 \mathrm{mg} / \mathrm{mL}$.

To validate the model predictions, a 3 -stage SPTFF with $88 \mathrm{~cm}^{2}$ membranes in series was first conducted with a feed concentration of $8.2 \mathrm{mg} / \mathrm{mL}$ at feed fluxes of $116 \mathrm{LMH}$ and $68 \mathrm{LMH}$. The output concentration measured from each stage is shown in overlay with the model predictions in Figure 4. The stagnant film model with the simplified expression for $\mathrm{k}(\mathrm{Q})$ derived from the Sherwood number was observed to work well within the range of feed fluxes that the model was built on, i.e., $34-136 \mathrm{LMH}$. The model could also be used to accurately predict the output concentration of different membrane configurations at different feed flow rates and concentrations within this range.

\section{Model Prediction at Low Flow Rates}

Accuracy of the model at lower feed fluxes relevant to a continuous process was then investigated. As shown in Figure 4, an exponential increase was observed in the model output concentration at the first stage, around $25 \mathrm{LMH}$. This inaccuracy was observed to prevail at low feed fluxes and high conversions. This phenomenon is further demonstrated in Figure 5A, where predicted conversion and concentration factors at low flow rates are compared. High conversions of about 0.85 and above occurred at low flux and feed concentrations $\left(\mathrm{C}_{\mathrm{f}}\right)$ into a single stage. These conditions led to a high degree of membrane polarization $\left(\mathrm{C}_{\mathrm{w}} / \mathrm{C}_{\mathrm{b}}\right)$, which in turn increased the predicted permeate flux (Equation. 3.7) to be greater than the feed flux (Q) - which is physically erroneous due to conversion values $>1$ (Equation 3.7 ).

Figure 5B shows the predicted conversion as a function of feed flux at different input concentrations. The 
conversion was observed to be greater than 1 at a flux of $20 \mathrm{LMH}$ at a $\mathrm{C}_{\mathrm{f}}$ of $4 \mathrm{mg} / \mathrm{mL}$, whereas at $\mathrm{C}_{\mathrm{f}}$ of 15 $\mathrm{mg} / \mathrm{mL}$ it did not exceed the same until it reached $5 \mathrm{LMH}$. This observation clarified the fact that low feed concentrations led to a higher polarization term. However, the effect of the polarization on the permeate flux calculation is diminished by the natural log operation. Therefore, it is likely that this sharp increase in conversion at low feed fluxes is due to an overestimation of the mass transfer coefficient. However, these predictions were extrapolated outside the experimental conditions used to build the model, as the lowest feed flux tested was only $34 \mathrm{LMH}$. Therefore, additional flux excursion experiments were performed at 7.2 and 18.7 LMH to account for behavior at lower feed fluxes (Figure 6).

Upon incorporation of additional low flow rate data into the model, the prediction of the mass transfer coefficient was not significantly affected (Fig. 6B). However, large increases in $\mathrm{C}_{\mathrm{w}}$ were seen at low flow rates thereby further increasing the polarization term and decreasing the accuracy of the model (Fig. 6C). This data confirms that the original model was not inaccurate due to a lack of data points within the design space but that it is only accurate for moderate to small values of $\mathrm{C}_{\mathrm{w}} / \mathrm{C}_{\mathrm{b}}$. This confirms the constraint on polarization concentration values for the stagnant film model as previously reported (Zydney, 1997). At these low flow rates, the estimation of $\mathrm{k}$ and $\mathrm{C}_{\mathrm{w}}$ as a function of flow rate alone is no longer appropriate.

To corroborate this notion, a series of experiments were conducted at decreasing feed fluxes to determine the maximum possible conversion for a single feed concentration. A feed flux range of 5 to $60 \mathrm{LMH}$ was investigated across one $88 \mathrm{~cm}^{2}$ membrane with a feed concentration of $5.45 \mathrm{mg} / \mathrm{mL}$. These results are shown in Figure 7. The model fits the experimental data accurately above $20 \mathrm{LMH}$. Below this point, the modeled conversion continued to exponentially increase while the experimental conversion plateaued. However, the experimental output concentration continued to increase significantly, approaching the theoretical maximum $\mathrm{C}_{\mathrm{w}}$ for this feed concentration. This was possibly due to small increases in conversion resulting in large increases in output concentration at low fluxes. For example, at these experimental conditions, the difference between a conversion of 0.96 and 0.97 is an output concentration of 135 and $180 \mathrm{mg} / \mathrm{mL}$ respectively. Thus, output concentration for low feed concentration systems was seen to be very sensitive to flow rates below $20 \mathrm{LMH}$. Therefore, any small errors in predicted conversion led to vast changes in predicted concentration. The conversion plateau seen in Fig 7A could not be predicted by the stagnant film model. Furthermore, the nature of the plateau would likely vary with feed concentration. For example, at a feed concentration of $15 \mathrm{mg} / \mathrm{mL}$ in this experiment, the maximum output concentration would be around $390 \mathrm{mg} / \mathrm{mL}$, which is not achievable in practice. To accurately characterize the plateau behavior and improve the accuracy of the model at low feed fluxes, this experiment would need to be repeated with a range of feed concentrations. This data could be used in combination with stagnant film modeling at higher feed fluxes to extend the range of prediction.

\section{Limitations of Stagnant Film Model}

Applying certain corrective measures to the inaccuracies of $\mathrm{k}$ and $\mathrm{C}_{\mathrm{w}}$ is challenging due to the interdependency of both these parameters. Firstly, $\mathrm{C}_{\mathrm{w}}$ cannot be easily or reliably measured directly, and our data suggests that it varies with the feed flux and is capable of increasing the error margins of model predictions. Secondly, deriving $k$ from Sherwood number (Equation 3.5) encompasses $\mathrm{C}_{\mathrm{w}}$ information and also includes terms that are difficult to measure accurately, such as diffusivity.

As an exercise, it was assumed that $k$ was accurately deduced from the slope of the $J \operatorname{vs} \ln \left(C_{b}\right)$ graph. $C_{w}$ was then calculated by rearranging Equation 3.3 into Equation 4.2 and solving for $\mathrm{J}$ from the experiment in Figure 7. This approach was inspired by van Reis et al. who combined the stagnant film and osmotic pressure models to create a constant $\mathrm{C}_{\mathrm{w}}$ ultrafiltration process control (van Reis et al., 1997).

$C_{w}=C_{b} * \exp \left(\frac{J_{\exp }}{k_{\text {model }}}\right)(4.2)$

Results of this analysis are shown in Figure 8A. According to this calculation, the $\mathrm{C}_{\mathrm{w}}$ at $5 \mathrm{LMH}$ was 59 $\mathrm{mg} / \mathrm{mL}$. This could not have been the case since the output concentration at this flux was measured to be $112 \mathrm{mg} / \mathrm{mL}$ (Figure 7B). 
Conversely, the measured wall concentrations from the flux excursion experiments were assumed to be true. In other words, it was assumed that $\mathrm{C}_{\mathrm{w}}$ could be accurately derived from the $\mathrm{x}$-intercepts of the $\mathrm{J}$ vs $\ln \left(\mathrm{C}_{\mathrm{b}}\right)$ graph (Figure 6). $\mathrm{k}$ was then calculated by rearranging equation 3.3 into equation $4.3 \mathrm{using}$ the flux values determined in Figure 7.

$k=\frac{J_{\exp }}{\ln \left(\frac{C_{w, m o d e l}}{C_{b}}\right)}$

The results of this analysis are shown in Figure 8B. This calculation supports the idea that the method for calculating $\mathrm{k}$ with the simplified model (equation 3.6) overpredicts $\mathrm{k}$ at low flow rates. These exercises reinstated the higher accuracy of the stagnant film model at high fluxes, which decreased as the low flow rate regime was entered.

\section{Sherwood Number Approximation}

Thus far, we worked with a simplified equation for $\mathrm{k}$ based on equation 3.6, allowing us to neglect the effects of concentration-dependent terms, such as viscosity and diffusivity. This approach only granted our model accuracy down to about $25 \mathrm{LMH}$. With the large range of calculated wall concentrations, it is not valid to assume that fluid properties remain the same. Specific to our operating regime, $\mathrm{C}_{\mathrm{w}}$ likely has a more complex dependency on other operating parameters. Therefore, at low flow rates, we hypothesize that $\mathrm{k}$ would be a function of the bulk fluid properties as well.

To investigate this phenomenon, a viscosity curve for $\mathrm{mAb} A$ was generated as a function of concentration up to $260 \mathrm{mg} / \mathrm{mL}$ (Figure 9). An exponential increase in viscosity with increasing concentration was observed as expected. At low feed fluxes, highly concentrated protein at the membrane wall can be imagined to be very viscous compared to the bulk solution. However, producing sufficient protein at high enough concentrations to mimic wall concentration is nearly impossible. Even if it could be done, then the accuracy of the viscosity measurements would be the limiting factor. Additionally, mass transfer coefficient calculations often leave out the dependence of the diffusion coefficient on concentration (Foley, 2013). High concentration and viscosity at the surface of the membrane ultimately affect the diffusivity of the protein. Therefore, the diffusivity term used in the Sherwood number calculation should be a function of concentration. However, measuring diffusivity would have the same limitations as viscosity.

Furthermore, even if these terms could be accurately measured, they rely on knowing the value of $\mathrm{C}_{\mathrm{w}}$ at a given feed flux and concentration, which is difficult to measure or estimate without already knowing k. Calculating the mass transfer coefficient to develop a predictive model using the complete Sherwood number can be tedious. Alternatively, data-based empirical approaches can be adapted to predict these concentrations with greater accuracy than first principles-based approaches. However, such models would need to be restricted to similar experimental conditions, setups, and process parameters to achieve a low degree of error on the predictions.

\section{Conclusions}

The stagnant film model has been widely used to describe the behavior of filtrate flux and mass transfer for the ultrafiltration of biopharmaceuticals (Foley, 2013; Teske et al., 2010; Zydney, 1997). In this work, we demonstrate that the model in description was observed to be highly accurate down to a feed flux of about 25 $\mathrm{LMH}$, below which it could no longer accurately predict the system's behavior. A potential explanation for the lack of predictability of the model arises from the use of a simplified version of the Sherwood number to estimate the mass transfer coefficient as a function of feed flux. This common simplification assumes constant fluid properties in the bulk and at the membrane surface. Additionally, the mass transfer coefficient also serves as a lumped parameter that captures the properties of the membrane as well as the protein. However, since the wall concentration and viscosity vary so widely across feed flux, this assumption could lead to the discrepancies we see with the model predictions. Thus, the model fails at low feed fluxes and feed concentrations due to high membrane polarization and a lack of accurate understanding of the effects of each of these variables on each other - and the system - at these conditions. 
In order to utilize the Sherwood number to accurately calculate the mass transfer coefficient, viscosity and diffusivity must be determined as a function of concentration. These values are difficult to accurately measure and can introduce significant errors in the calculation. In addition, $\mathrm{C}_{\mathrm{w}}$ must be known in order to calculate viscosity at the wall. Determining $\mathrm{C}_{\mathrm{w}}$ using the stagnant film model negates any effect of feed concentration. Furthermore, estimating $\mathrm{C}_{\mathrm{w}}$ by rearranging the stagnant film model equation requires very accurate estimates for $\mathrm{k}$. Thus, even if the concentration-dependent terms could be measured accurately, the complete Sherwood calculation depends on knowing $\mathrm{C}_{\mathrm{w}}$.

Another possible explanation could be the fact that the stagnant film theory, equation 3.1, which is derived from Fick's second law, neglects the axial diffusion terms to derive a finite solution to the following differential equation:

$V_{x} \frac{\partial C}{\partial x}+V_{z} \frac{\partial C}{\partial z}=D\left[\frac{\partial^{2} C}{\partial x^{2}}+\frac{\partial^{2} C}{\partial z^{2}}\right](5.1)$

where $\mathrm{x}$ is the direction of axial diffusion and $\mathrm{z}$ is the direction of convective transfer. To provide an analytical solution to this equation, axial diffusion and the velocity distribution in the $\mathrm{x}$ direction are neglected (Miranda \& Campos, 2002). However, it also needs to be taken into consideration that at high conversion observed at lower feed flow rates the bulk concentration increases and the velocity decreases significantly along the length of the membrane (H. Lutz, 2015). It can be hypothesized that axial diffusion becomes significant and should not be neglected in eq. 5.1. The contribution of both axial and convective mass transfer can be a possible explanation to the plateauing of the conversions at low fluxes, as their consideration would result in lower concentration factors than predicted by the stagnant film model. Lastly, throughout steady state, concentration gradients within the physical system of operation, protein - protein interactions, buffer effects and specific geometries of membranes on the concentration product may impact the relationship between variables used in to build the model, which have not been considered in our studies. Jabra et. al, considered such variables to model and predict output concentrations within a different set of inlet flow rates (Jabra et al., 2021). While accounting for system variations at low flow rates such as the range in question within this paper is possible, doing so would require a battery of additional studies and severely increase the complexity of the model.

In summary, the stagnant film model can be used to accurately predict the output concentration of SPTFF processes at reasonable feed fluxes, which in this work were above $25 \mathrm{LMH}$. This facilitates proper membrane sizing and configuration for a wide range of processes with minimal additional experimental work to perform. This is particularly attractive for SPTFF based ultrafiltration in continuous biomanufacturing where a target product concentration must be achieved under feed flow rate and concentration constraints resulting from upstream unit operations. Therefore, this model can be used to design membrane configurations capable of achieving a target concentration across a range of feed concentrations and flow rates with minimal process development. However, we show that the typical assumptions made to utilize this model fail at combinations of low feed fluxes and concentrations. In this regime, there appears to be a more complex relationship between the mass transfer coefficient, wall concentration, feed concentration, and flow rate, than the model can currently capture. At lower fluxes, an empirical characterization of the design space or alternative modeling approaches can be utilized to continue achieving the benefits of model-based concentration prediction.

\section{Acknowledgements}

The authors would like to acknowledge Megan Le for executing viscosity measurements, Bryan Chan, Nidhi Thite, and Sushmitha Krishnan for SPTFF development efforts, and Antonio Ubiera for providing a review of the manuscript.

\section{Conflict of Interest}

None

\section{References}

Dizon-Maspat, J., Bourret, J., D’Agostini, A., \& Li, F. (2012). Single pass tangential flow filtration to 
debottleneck downstream processing for therapeutic antibody production. Biotechnology and Bioengineering , 109 (4), 962-970. https://doi.org/10.1002/bit.24377

Foley, G. (2013). Membrane filtration: a problem solving approach with MATLAB . Cambridge University Press.

Jabra, M. G., Lipinski, A. M., \& Zydney, A. L. (2021). Single Pass Tangential Flow Filtration (SPTFF) of monoclonal antibodies: Experimental studies and theoretical analysis. Journal of Membrane Science , 637 , 119606. https://doi.org/https://doi.org/10.1016/j.memsci.2021.119606

Lutz, H. (2015). 2 - Membranes (H. B. T.-U. for B. Lutz (ed.); pp. 7-30). Woodhead Publishing. https://doi.org/https://doi.org/10.1016/B978-1-907568-46-6.00002-1

Millipore. (1999). Protein concentration and diafiltration by tangential flow filtration. TB032 Rev:B. https://www.bio-link.org/sites/files/tff_technical_brief.pdf

Miranda, J. M., \& Campos, J. B. L. M. (2002). Mass transfer in the vicinity of a separation membrane - the applicability of the stagnant film theory. Journal of Membrane Science , 202 (1), 137150. https://doi.org/https://doi.org/10.1016/S0376-7388(01)00747-5

Teske, C. A., Lebreton, B., \& van Reis, R. (2010). Inline ultrafiltration. Biotechnology Progress , 26 (4), 1068-1072. https://doi.org/10.1002/btpr.404

Thakur, G., \& Rathore, A. S. (2021). Modelling and optimization of single-pass tangential flow ultrafiltration for continuous manufacturing of monoclonal antibodies. Separation and Purification Technology, 276 , 119341. https://doi.org/https://doi.org/10.1016/j.seppur.2021.119341

van Reis, R., Goodrich, E. M., Yson, C. L., Frautschy, L. N., Whiteley, R., \& Zydney, A. L. (1997). Constant Cwall ultrafiltration process control. Journal of Membrane Science , 130 (1), 123-140. https://doi.org/https://doi.org/10.1016/S0376-7388(97)00012-4

Zydney, A. L. (1997). Stagnant film model for concentration polarization in membrane systems. Journal of Membrane Science, 130 (1), 275-281. https://doi.org/https://doi.org/10.1016/S0376-7388(97)00006-9

\section{Abbreviations:}

\begin{tabular}{|c|c|}
\hline Symbol & Interpretation \\
\hline $\mathbf{J}$ & Permeate flux $\left(\mathrm{L} / \mathrm{m}^{2} / \mathrm{hr}\right.$ or $\left.\mathrm{LMH}\right)$ \\
\hline D & Diffusivity of $\mathrm{mAb} A$ in the buffer $\left(\mathrm{m}^{2} / \mathrm{s}\right)$ \\
\hline$\delta$ & Thickness of the gel layer \\
\hline $\mathrm{C}_{\mathrm{w}} / \mathrm{C}_{\mathrm{f}} / \mathrm{C}_{\mathrm{b}} / \mathrm{C}_{\mathrm{p}} / \mathrm{C}_{\mathrm{r}}$ & Solute concentration at wall/feed/bulk/permeate/retentate \\
\hline $\mathbf{P}($ Feed/Retentate/Permeate $)$ & PMAT Pressures at respective inlets / outlets \\
\hline Sh & Sherwood Number \\
\hline $\operatorname{Re}$ & Reynolds Number \\
\hline Sc & Schmidt Number \\
\hline $\mathbf{L}$ & Length of the membrane \\
\hline$d_{h}$ & hydraulic diameter \\
\hline$\mu_{\beta} / \mu_{\omega}$ & Viscosity of the bulk solution / wall concentration \\
\hline$\beta, \alpha, \beta, \varsigma, \zeta$ & Empirical Constants \\
\hline A & Membrane area $\left(\mathrm{m}^{2}\right)$ \\
\hline $\mathrm{Q} / \mathbf{q}_{\mathbf{f}}$ & Feed flux $(\mathrm{LMH})$ / Feed flow rate $(\mathrm{L} / \mathrm{hr})$ \\
\hline$?$ & Conversion (fraction of flow exiting through the permeate) \\
\hline $\mathrm{k}_{0} / \mathrm{k}_{1}$ & Known and unknown mass transfer coefficient at feed flow rates $\mathrm{q}_{0}$ and $\mathrm{q}_{1}(\mathrm{~L} / \mathrm{hr})$ res \\
\hline $\mathbf{k}_{\text {model }}$ & Defined by equation 3.6 using values from the slopes of $\mathrm{J}$ vs $\ln \left(\mathrm{C}_{\mathrm{b}}\right)$ (Fig. 6A) \\
\hline $\mathrm{C}_{\mathrm{w}, \text { model }}$ & Power regression fit of the $\mathrm{x}$-intercepts on $\mathrm{J}$ vs $\ln \left(\mathrm{C}_{\mathrm{b}}\right)$ \\
\hline $\mathbf{J}_{\text {exp }}$ & Measured experimental permeate flux (Figure 7) \\
\hline
\end{tabular}


Tables:

\begin{tabular}{ll}
\hline Flow Rate $\left(\mathrm{L} / \mathrm{m}^{2} \mathrm{hr}, \mathrm{LMH}\right)$ & $136,82,34,18.7,7.2$ \\
Transmembrane Pressure $(\mathrm{psi})$ & $5,10,15,20,25$ \\
Input Protein Concentration $(\mathrm{mg} / \mathrm{mL})$ & $4,20,40,60,90$ \\
\hline
\end{tabular}

Table 1: Design space for flux excursion experiments

Figures Legends:

Figure 1: Flux excursion data presented as permeate flux $(\mathrm{J})$ vs. TMP by feed flow rate and concentration

Figure 2: Permeate flux versus feed concentration at a TMP of 15psi for three different flow rates, where k $=-\mathrm{m}$ and $\mathrm{C}_{\mathrm{w}}=\mathrm{x}$-intercept

Figure 3: Effect of feed flow rate $(\mathrm{Q})$ on the mass transfer coefficient $(\mathrm{k})$ and wall concentration $\left.\left(\mathrm{C}_{\mathrm{w}}\right) \mathrm{A}\right) \mathrm{k}$ vs $Q$ fit by the simplified Sherwood number in equation 3.6 and B) $C_{w}$ vs Q fit linearly by $\mathrm{mQ}+\mathrm{b}$

Figure 4: Predicted output concentration versus feed flux by stages of equal surface area with overlaid experimental data obtained at feed fluxes of 68 and $116 \mathrm{LMH}$

Figure 5: Stagnant film modeling at low flow rates and feed concentrations A) predicted conversion and concentration versus feed flux, and B) predicted conversion versus feed flux by feed concentration

Figure 6: Stagnant film modeled parameters with additional flux excursion data performed at 7.2 and 18.7 LMH A) Permeate flux versus feed concentration at a TMP of 15 psi for five different feed flow fluxes, B) $\mathrm{k}$ vs $\mathrm{Q}$ fit with equation 3.6 for the full range of flux excursion data (7.2-136 LMH, dashed line) vs only the higher fluxes (34-126LMH, solid line) and C) $\mathrm{C}_{\mathrm{w}}$ vs Q fit with a linear regression for 34-126LMH (solid line) vs a power regression for 7.2-136 LMH (dashed line)

Figure 7: Experimentally determined vs stagnant film modeled conversion (A) and output concentration (B) vs feed flux of a one membrane system with a feed concentration of $5.4 \mathrm{mg} / \mathrm{mL}$

Figure 8: (A) $\mathrm{C}_{\mathrm{w}}$ calculated according to equation 4.2 with the experimentally determined permeate flux from the experiment shown in Fig.7 and $\mathrm{k}$ from the slope of the $\mathrm{J}$ vs $\ln \left(\mathrm{C}_{\mathrm{b}}\right)$ graph, (B) k calculated according to equation 4.3 the experimentally determined $\mathrm{J}$ from the experiment shown in Fig.7 and $\mathrm{C}_{\mathrm{w}}$ from the $\mathrm{x}-$ intercept of the $\mathrm{J}$ vs $\ln \left(\mathrm{C}_{\mathrm{b}}\right)$ graph

Figure 9: Trend of mAb Viscosity as a function of mAb concentration 